\title{
Guest-editorial
}

\section{Special issue on Knowledge-based Modes of Human-Computer Interaction}

\author{
Maria Virvou and George A. Tsihrintzis* \\ Department of Informatics, The University of Piraeus, 80, Karaoli \& Dimitriou St., 18534 Piraeus Greece
}

This special issue on "Knowledge-based Modes of Human-Computer Interaction" aims at presenting some novel ways and relevant challenging issues of how users interact with computers in knowledge-based environments. As computer users are spreading and include people of all ages, backgrounds, professions, education levels, aims, profiles, preferences and personalities, human-computer interaction has to undertake the difficult task of personalization, adaptivity, virtual reality presentation, intelligent multimedia interaction and so on. The special issue focuses on both theoretical issues as well as applications of intelligent user interfaces within the area of human-computer interaction including methodologies, design environments, user interface development life cycle, empirical studies, multimedia/multimodal signal processing and evaluation.

We have received a large number of submissions to the special issue. Each submitted paper was reviewed by at least two independent reviewers for novelty and clarity of the research reported in it. Additionally, as guest co-editors, we looked over all the manuscripts. For inclusion in the special issue, we have selected six papers from those submitted.

The first four papers describe novel multimodal ways of human-computer interaction. Specifically, the first paper, authored by Alepis and Virvou, is on "Object Oriented Architecture for Affective Multimodal eLearning Interfaces." The second paper, authored by
Alotaibi and Rigas, is on "An Empirical Approach to Customer Knowledge Management." The third paper, authored by Odya, Czyzewski, Grabkowska, and Grabkowski, is on "Smart Pen - A New Multimodal Computer Control Tool for Graphomotorical Therapy." The fourth paper, authored by Patsakis and Alexandris, is on "Enhancing 2D GUIs with 3D Input Devices."

The next two papers present novel multimedia signal processing methodologies, which may prove efficient in current and future knowledge-based modes of human-computer interaction. Specifically, the fifth paper, authored by Kunka, Kostek, Kulesza, Szczuko, and Czyzewski, is on "Gaze-Tracking-Based Audio-Visual Correlation Analysis Employing Quality of Experience Methodology." Finally, the sixth paper, authored by Lampropoulou, Lampropoulos, and Tsihrintzis is on "Music Genre Classification based on Ensemble of Signals produced by Source Separation Methods."

The guest co-editors would like to thank Profs.-Drs. Lakhmi C. Jain and Gloria Phillips-Wren, Chief Editors of the Intelligent Decision Technologies Journal, for agreeing to the publication of the special issue on "Knowledge-based Modes of Human-Computer Interaction." The guest co-editors would also like to thank all the authors for their contributions, the reviewers for their time and effort in reviewing manuscripts, and the journal production team for their support and help in producing the special issue.

\footnotetext{
*Corresponding author. E-mail: geoatsi@unipi.gr.
} 